\title{
Transcatheter retrieval of an Amplatzer Vascular Plug
}

\author{
Yozo Sato ${ }^{1 A, B, E, F}$, Yoshitaka Inaba ${ }^{1 B, E}$, Takaaki Hasegawa ${ }^{1 B}$, Shinichi Murata ${ }^{1 B}$, Yuki Kimbara ${ }^{1 B}$, Yoshiki Senda ${ }^{2 B}$ \\ 'Departments of Diagnostic and Interventional Radiology, Aichi Cancer Centre Hospital, 1-1 Kanokoden, Chikusa-ku, Nagoya, Japan \\ 2Departments of Gastroenterological Surgery, Aichi Cancer Centre Hospital, 1-1 Kanokoden, Chikusa-ku, Nagoya, Japan
}

\section{Abstract}

Purpose: An Amplatzer Vascular Plug (AVP), which was designed as a permanent occluding device derived from the Amplatzer Septal Occluder and Amplatzer Duct Occluder, is a useful embolic device that can be precisely deployed in medium to large vessels with high resistance to migration. However, migration of these Amplatzer devices has been reported as a relatively rare but major complication.

Case report: A 59-year-old woman was referred for the treatment of advanced pancreatic body cancer; after systemic chemotherapy, distal pancreatectomy with en bloc celiac axis resection (DP-CAR) was planned as curative treatment. Therefore, preoperative embolisation of the common hepatic artery (CHA) for arterial redistribution was performed. Although a 6-mm AVP II was deployed at the mid-portion of the CHA, the AVP migrated to the proper hepatic artery. Although migrated AVP retrieval using a goose neck snare was attempted, it was impossible to retrieve it into the 5-F guiding sheath. Therefore, the AVP was delivered to the splenic artery, which was planned to be resected in DP-CAR. Finally, a 10-mm AVP II was redeployed at the proximal portion of the CHA, and complete occlusion was achieved.

Conclusions: When AVP retrieval is not possible, delivery to the other arteries having lesser influence might be an alternate technique.

Key words: embolisation, therapeutic/methods, septal occluder device, preoperative care.

\section{Introduction}

An Amplatzer Vascular Plug (AVP; Abbott Vascular, Santa Clara, California) is a useful embolic device that can be precisely deployed in medium to large vessels with high resistance to migration [1]. The AVP has developed from a single device to a group of four models (AVP, AVP II, AVP III, and AVP 4) that can be used for various types of anatomy, haemodynamics, and lesion entities. The original AVP was designed as a permanent occluding device derived from the Amplatzer Septal Occluder and Amplatzer Duct Occluder [1]. These Amplatzer devices have a self-expanding feature with sufficient radial force on the vessel wall to minimise movement; however, migra- tion of the Amplatzer occluder in cardiac interventions has been reported as a relatively rare but major complication [2-4].

\section{Case report}

This case report of transcatheter retrieval of AVP migration to the proper hepatic artery was exempt from institutional review board approval. A 59-year-old woman was referred for the treatment of advanced pancreatic body cancer with paraaortic lymph node metastases. Enhanced computed tomography revealed shrinkage of primary tumour and lymph node metastases after systemic chemotherapy. No abnormal uptake of lesions in

\section{Correspondence address:}

Yozo Sato, Department of Diagnostic and Interventional Radiology, Aichi Cancer Centre Hospital, 1-1 Kanokoden, Chikusa-ku, Nagoya 464-8681, Japan,

phone: +81-52-762-6111, fax:+81-52-764-2966, e-mail: ysato@aichi-cc.jp

Authors' contribution:

A: Study design, B: Data collection, C: Statistical analysis, D: Data interpretation, E: Manuscript preparation, F: Literature search, G: Funds collection 

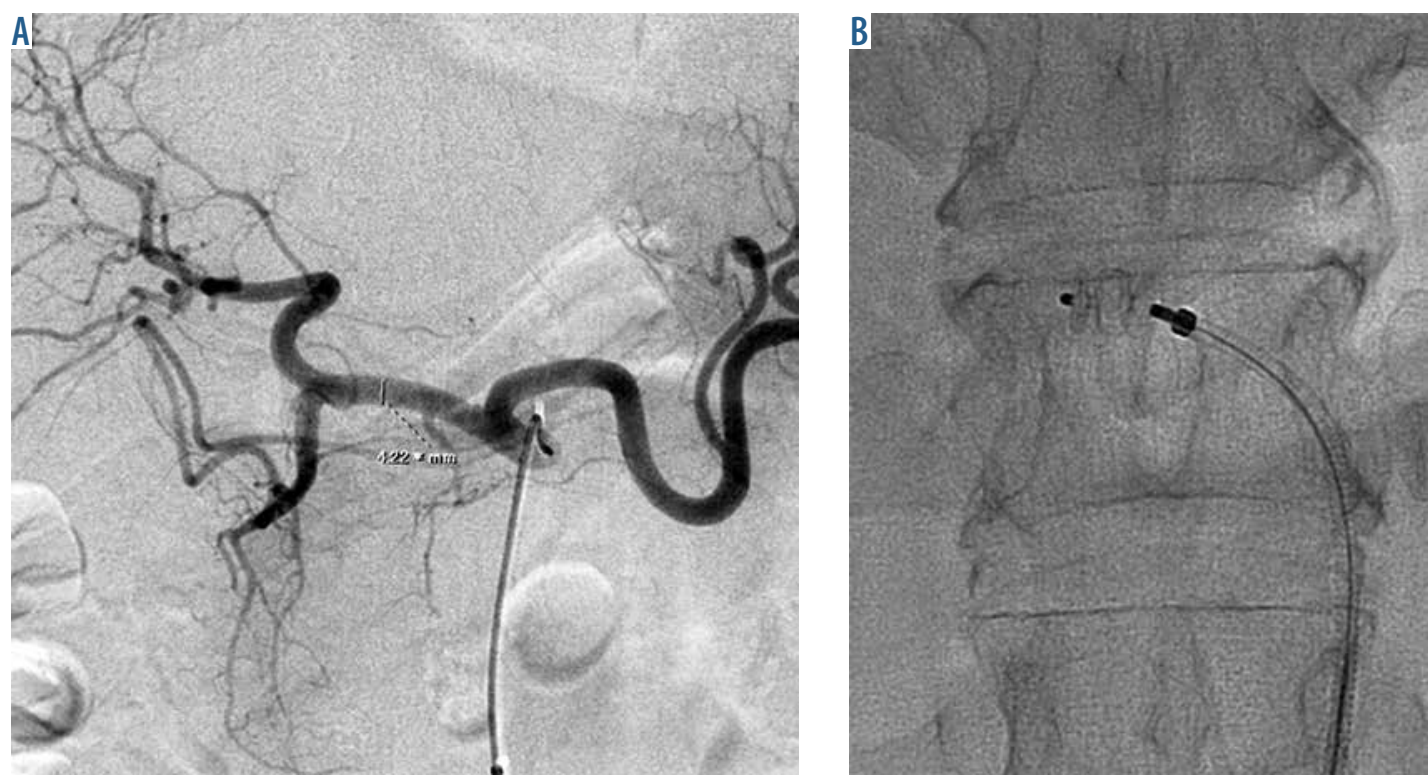

Figure 1. A) Celiac arteriography reveals the common hepatic artery (CHA) with a maximum diameter of 4.2 mm. B) A 6-mm AmplatzerVascular Plug (AVP) II is deployed at the mid-portion of the CHA

$\left[{ }^{18} \mathrm{~F}\right]$ fluorodeoxyglucose-positron emission tomography/ computed tomography (FDG-PET/CT) was observed. After seven cycles of systemic chemotherapy, distal pancreatectomy with en bloc celiac axis resection (DP-CAR) was planned as curative treatment. Therefore, preoperative embolisation of the common hepatic artery (CHA) for arterial redistribution was employed to prevent postoperative ischaemic complications.

Angiography using a 4-F diagnostic catheter revealed the left gastric artery (LGA) arising from the aorta and the left hepatic artery arising from the LGA. Celiac arteriography revealed $\mathrm{CHA}$ with a maximum diameter of $4.2 \mathrm{~mm}$ (Figure 1A); a 4-F catheter was advanced to the gastroduodenal artery. A 5-F guiding sheath (Flexor ${ }^{\circ}$ Ansel; Cook Medical, Bloomington, Indiana) was advanced to the $\mathrm{CHA}$ along with a $4-\mathrm{F}$ catheter, and a $6-\mathrm{mm}$ AVP II was deployed at the mid-portion of the CHA (Figure 1B). Although the AVP was completely expanded, it did not move, even if force was exerted on the delivery cable. However, the AVP migrated to the proximal portion of the proper hepatic artery approximately 30 seconds after detachment (Figure 2A). Although migrated AVP retrieval using a goose neck snare (Amplatz GooseNeck Snare; Medtronic, Minneapolis, Minnesota) was attempted, it was impossible to retrieve it into the 5-F guiding sheath because the AVP delivery screw became stuck within the sheath edge (Figures $2 \mathrm{~B}-\mathrm{C}$ ). However, the guiding sheath with snaring the AVP was gradually pulled to the proximal side and the guiding sheath could be directed to the splenic artery. Therefore, the AVP was delivered to the splenic artery, which was planned to be resected in DP-CAR. Finally, a 10-mm AVP II was redeployed at the proximal portion of the $\mathrm{CHA}$, and complete occlusion was achieved (Figures 3A-B). The distal portion of the splenic artery was visualised via the collateral blood flow from the LGA.
Although mild-spleen infarction occurred, DP-CAR was successfully performed three days after embolisation.

\section{Discussion}

The utility of CHA preoperative embolisation for DPCAR has been reported [5,6]; AVP, which has a low risk of migration, can be an optimal embolic device. The incidence of device migration (including an Amplatzer Septal Occluder) associated with transcatheter occlusion of atrial septal defects was reported to be from $0.55 \%$ to $3.5 \%$ $[2,3]$. The commonest cause of migration was the use of an undersized device. AVP II was completely expanded due to an undersized device after deployment in the case presented here; hence, the AVP should have been retrieved before detachment. Although transcatheter retrieval using a goose neck snare and/or a basket catheter was recommended, the technical success rate was reported to be from $50 \%$ to $75 \%$, and surgical retrieval was required in failure cases [2-4]. In the present case, the migrated AVP could be snared; however, it was impossible to retrieve it into the 5-F guiding sheath. Because there was no larger diameter sheath at that time, there was no other option but to deliver the AVP to the splenic artery, which was planned to be resected in DP-CAR. It may be essential to prepare larger-sized sheaths that enable AVP retrieval without it becoming stuck in the delivery screw. However, when AVP retrieval is not possible, delivery to the other arteries having lesser influence might be an alternate technique.

\section{Conclusions}

This report presented a case of transcatheter retrieval of AVP migration to the proximal portion of the proper hepatic artery. Although migrated AVP retrieval using 

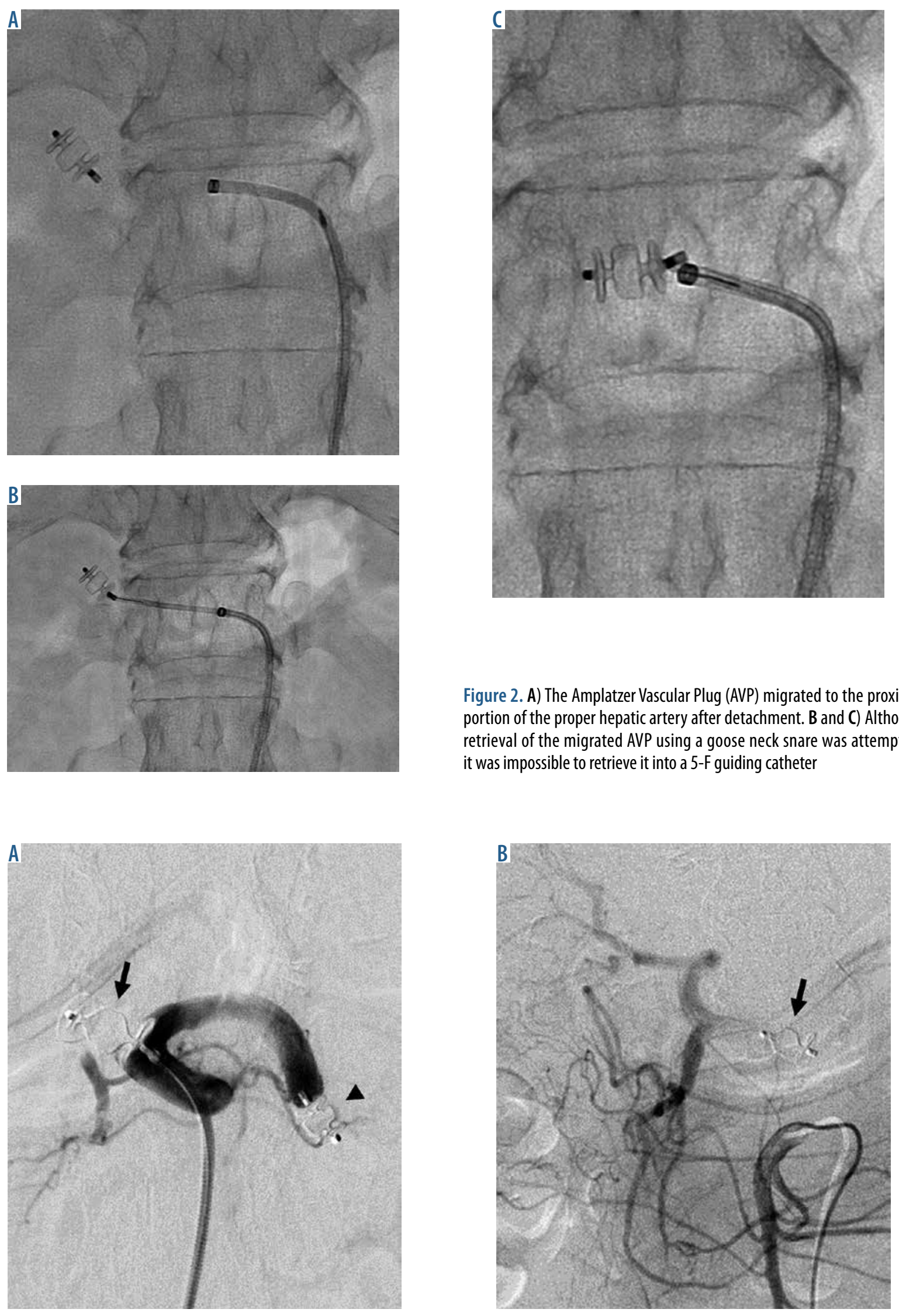

Figure 2. A) The Amplatzer Vascular Plug (AVP) migrated to the proximal portion of the proper hepatic artery after detachment. B and C) Although retrieval of the migrated AVP using a goose neck snare was attempted, it was impossible to retrieve it into a $5-\mathrm{F}$ guiding catheter

Figure 3. A) A 6-mm Amplatzer Vascular Plug (AVP) (arrow head) was delivered to the splenic artery and a 10-mm AVP II (arrow) was redeployed at the proximal portion of the common hepatic artery (CHA). B) Superior mesenteric arteriography reveals good blood flow to the intrahepatic artery via the pancreatic arterial arcade 
a goose neck snare was attempted, it was impossible to retrieve it into the 5 -F guiding sheath. Therefore, the AVP was delivered to the splenic artery, which was planned to be resected in DP-CAR. When AVP retrieval is not possible, delivery to the other arteries having lesser influence might be an alternate technique.

\section{References}

1. Wang W, Li H, Tam MD, et al. The amplatzer vascular plug: a review of the device and its clinical applications. Cardiovasc Intervent Radiol 2012; 35: 725-740.

2. Moore J, Hegde S, El-Said H, et al. Transcatheter device closure of atrial septal defects: a safety review. JACC Cardiovasc Interv 2013; 6: 433-442.

3. Chessa M, Carminati M, Butera G, et al. Early and late complications associated with transcatheter occlusion of secundum atrial septal defect. J Am Coll Cardiol 2002; 39: 1061-1065.

\section{Conflict of interest}

The authors report no conflict of interest.

4. Goel PK, Kapoor A, Batra A, et al. Transcatheter retrieval of embolized AMPLATZER Septal Occluder. Tex Heart Inst J 2012; 39: 653-656.

5. Takasaka I, Kawai N, Sato M, et al. Preoperative microcoil embolization of the common hepatic artery for pancreatic body cancer. World J Gastroenterol 2012; 18: 1940-1945.

6. Ishikawa M, Kajiwara K, Fukumoto W, et al. Blood Flow Redistribution Using the AMPLATZER Vascular Plug 4 before Distal Pancreatectomy with en Bloc Celiac Axis Resection. J Vasc Interv Radiol 2016; 27: 285-286 